\title{
Maximal Gauged Supergravity in Three Dimensions
}

\author{
H. Nicolai \\ Max-Planck-Institut für Gravitationsphysik, Mühlenberg 1, D-14476 Golm, Germany \\ H. Samtleben \\ LPT-ENS, * 24 Rue Lhomond, F-75231 Paris Cedex 05, France \\ (Received 11 October 2000; revised manuscript received 15 December 2000)
}

\begin{abstract}
We construct maximally supersymmetric gauged $N=16$ supergravity in three dimensions, thereby obtaining an entirely new class of anti-de Sitter supergravities. These models apparently cannot be derived from any known higher-dimensional theory and point to the existence of a new type of supergravity beyond $D=11$. One of their noteworthy features is a non-Abelian generalization of the duality between scalar and vector fields in three dimensions. Among the possible gauge groups, $\mathrm{SO}(8) \times \mathrm{SO}(8)$ is distinguished as the maximal compact gauge group, but there are also more exotic possibilities such as $F_{4(-20)} \times G_{2}$.

DOI: 10.1103/PhysRevLett.86.1686

PACS numbers: 11.10.Kk, 04.50. $+\mathrm{h}, 04.65 .+\mathrm{e}, 11.15 .-\mathrm{q}$
\end{abstract}

Gauged supergravities have attracted strong interest again recently in the context of the conjectured duality between anti-de Sitter (AdS) supergravities and superconformal quantum field theories on the AdS boundary [1]. Since the construction of maximal $N=8$ gauged supergravity in four dimensions [2] various maximal gauged supergravities in dimensions $D>4$ have been shown to exist [3]. Much less is known, however, about gauged supergravities in dimensions $D<4$ - despite the fact that the theories admitting $\mathrm{AdS}_{3}$ ground states are expected to be of particular interest for the AdS/conformal field theory (AdS/CFT) duality due to the rich and rather well understood structure of two-dimensional superconformal field theories. A better understanding of the supergravity side is of vital importance for any further test of this duality.

In this Letter, we construct maximally supersymmetric gauged $N=16$ supergravity in three dimensions. This is the most complex and most symmetric of all theories of this type known so far (and may remain so in view of the appearance of the maximal exceptional Lie group $E_{8(8)}$ in it). It gives rise to an entirely new class of threedimensional models, none of which is contained in or derivable by any known mechanism from the known supergravity or superstring theories in higher dimensions. This is all the more remarkable in view of the fact that these models constitute continuous deformations of a theory (ungauged $N=16$ supergravity $[4,5]$ ) that is obtained by a torus compactification of $D=11$ supergravity [6]. Their existence not only hints at a new type of supergravity beyond $D=11$ supergravity but also indicates that the framework of theories considered so far in attempts to unify string theories at the nonperturbative level may be inadequate. Likewise, the boundary theory associated with our $\mathrm{SO}(8) \times \mathrm{SO}(8)$ gauged supergravity is expected to enjoy an exceptional status among the superconformal field theories. The AdS/CFT correspondence indeed suggests a duality with a sector of maximally supersymmetric YangMills theory on the two-dimensional AdS boundarybut this is just matrix string theory, which itself has been proposed as a candidate for $M$ theory [7].

Topological gauged supergravities in three dimensions were constructed in [8]; these theories are supersymmetric extensions of Chern-Simons (CS) theories with $(p, q)$ supersymmetry and gauge groups $\mathrm{O}(p) \times \mathrm{O}(q)$ but have no propagating matter degrees of freedom. Matter coupled gauged supergravities can, of course, be obtained by direct dimensional reduction of gauged supergravities in $D \geq 4$ to three dimensions and below [9]; see also [10] and [11] for different constructions. However, no such theories preserving the maximal number of supersymmetries in three dimensions have been known until now.

The scarcity of results is in part explained by the different status of vector fields in three dimensions in comparison with their higher dimensional relatives, namely, their on-shell equivalence with scalar fields via the duality relation $\epsilon_{\mu \nu \rho} \partial^{\rho} \varphi^{m}=\partial_{[\mu} B_{\nu]}{ }^{m}$. This relation plays a special role in the derivation of maximal $N=16$ supergravity in three dimensions [4,5]: in order to expose its rigid $E_{8(8)}$ symmetry, all vector fields obtained by dimensional reduction of $D=11$ supergravity [6] on an 8-torus must be dualized into scalar fields. Thus an immediate (but, as we will see, only apparent) obstacle towards the gauging of the maximally extended three-dimensional supergravity is the a priori absence of vector fields that could be used for such a gauging. Let us therefore emphasize the main features by which our construction differs from previous ones:

(i) The theory makes simultaneous use of both scalar fields and their dual vectors, which appear via a CS term in order of the gauge coupling constant $g$ and therefore do not carry additional physical degrees of freedom. Their equations of motion yield a non-Abelian generalization of the standard duality relation between scalar and vector fields, 
such that a direct elimination of the latter is no longer possible (and would lead to a nonlocal theory in terms of the 128 physical scalar fields of $N=16$ supergravity).

(ii) Several consistent choices are possible for the gauge group $G_{0} \subset E_{8(8)}$, although $G_{0}=\mathrm{SO}(8) \times \mathrm{SO}(8)$ is distinguished as the maximal compact subgroup of $E_{8(8)}$ that can be made local. The noncompact gauge groups allowed by our construction do not arise as analytic continuations of a compact subgroup unlike the known noncompact gaugings in higher dimensions [12].

(iii) Conventional Kaluza-Klein compactification of $D=11$ (or $D=10$ ) supergravity cannot give rise to these gauged theories because it would yield Yang-Mills-type theories rather than non-Abelian CS theories and there are no 8-manifolds (or 7-manifolds) whose isometry groups would coincide with any of the possible gauge groups $G_{0}$.

(iv) Although the rigid $E_{8(8)}$ symmetry is broken, such that the gauged theory is only invariant under local $G_{0} \times$ $\mathrm{SO}(16)$, the construction makes essential use of properties of the exceptional Lie group $E_{8(8)}$. In particular, the check of local supersymmetry requires " $T$ identities" based on $E_{8(8)}$ analogous to the ones derived in [2].

The physical fields of ungauged maximal $N=16$ supergravity in three dimensions constitute an irreducible supermultiplet with 128 bosons and 128 fermions transforming as inequivalent fundamental spinors of $\mathrm{SO}(16)$ (see [5] whose conventions and notation we follow).

$$
\epsilon^{\mu \nu \rho}\left(B_{\nu \rho}{ }^{m}+2 \mathcal{V}^{m}{ }_{I J} \bar{\psi}_{\nu}^{I} \psi_{\rho}^{J}-2 i \Gamma_{A \dot{A}}^{I} \mathcal{V}^{m}{ }_{A} \bar{\psi}_{\nu}^{I} \gamma_{\rho} \chi^{\dot{A}}\right)=2
$$
whose compatibility condition gives rise to the equations of motion for the scalar fields. At the same time (4) serves to define the dual vector fields $B_{\mu}{ }^{m}$ with (Abelian) field strengths $B_{\mu \nu}{ }^{m}$. Although we are still concerned with Abelian vector fields at this point, we introduce special labels $m, n, \ldots$ for the generators of the gauge group $G_{0} \subset E_{8(8)}$ already here. The gauge group is properly described by means of its (symmetric) embedding tensor $\Theta_{\mathcal{A B}}$, such that

$$
B_{\mu}{ }^{m} t_{m} \equiv B_{\mu}^{\mathcal{A}} \Theta_{\mathcal{A B}} t^{\mathcal{B}} .
$$

We emphasize that the number of vector fields is not yet specified since it will depend on the choice of the gauge group $G_{0}$. Although the duality (4) holds only on-shell, we shall see that the gauged theory provides a natural off-shell framework which accommodates both the scalars and their dual vectors.

Under local supersymmetry the vectors transform as [14]

$$
\delta B_{\mu}{ }^{m}=-2 \mathcal{V}^{m}{ }_{I J} \bar{\epsilon}^{I} \psi_{\mu}^{I}+i \Gamma_{A \dot{A}}^{I} \mathcal{V}^{m}{ }_{A} \bar{\epsilon}^{I} \gamma_{\mu} \chi^{\dot{A}} .
$$

To gauge the theory, we now assume the existence of a suitable subgroup $G_{0} \subset E_{8(8)}$ with embedding tensor $\Theta_{\mathcal{A B}}$. The possible choices for $G_{0}$ are determined below by demanding consistency and full supersymmetry of
In addition, the theory contains the dreibein $e_{\mu}{ }^{\alpha}$ and 16 gravitino fields $\psi_{\mu}^{I}$. The scalar fields are described by an element $\mathcal{V}$ of the noncompact coset space $E_{8(8)} / \mathrm{SO}(16)$ in the fundamental 248-dimensional representation [4]. The equivalence of the fundamental and the adjoint representations of $E_{8(8)}$ is expressed by the relation

$$
\mathcal{V}^{-1} t^{\mathcal{A}} \mathcal{V}=\mathcal{V}^{\mathcal{A}}{ }_{\mathcal{B}} t^{\mathcal{B}}
$$

Indices $\mathcal{A}, \mathcal{B}, \ldots$ here label $E_{8(8)}$ generators $t^{\mathcal{A}}$ (see $[13,14]$ for our $E_{8(8)}$ conventions and some useful formulas). We split the generators $\left\{t^{\mathcal{A}}\right\}$ into 120 compact ones $\left\{X^{I J}\right\}$ and 128 noncompact ones $\left\{Y^{A}\right\}$.

$N=16$ supergravity is invariant under a hidden rigid $E_{8(8)}$ symmetry as well as a local $\mathrm{SO}(16)$ symmetry. The supersymmetry variations are [5]

$$
\begin{aligned}
\delta e_{\mu}{ }^{\alpha} & =i \bar{\epsilon}^{I} \gamma^{\alpha} \psi_{\mu}^{I}, & \mathcal{V}^{-1} \delta \mathcal{V} & =\Gamma_{A \dot{A}}^{I} \bar{\chi}^{\dot{A}} \epsilon^{I} Y^{A}, \\
\delta \psi_{\mu}^{I} & =D_{\mu} \epsilon^{I}, & \delta \chi^{\dot{A}} & =\frac{i}{2} \gamma^{\mu} \epsilon^{I} \Gamma_{A \dot{A}}^{I} P_{\mu}^{A} .
\end{aligned}
$$

The scalar fields couple to the fermions via the currents

$$
\mathcal{V}^{-1} \partial_{\mu} \mathcal{V}=\frac{1}{2} Q_{\mu}^{I J} X^{I J}+P_{\mu}^{A} Y^{A}
$$

where the composite $\mathrm{SO}(16)$ connection $Q_{\mu}^{I J}$ enters the covariant derivative $D_{\mu}$ in (2) and (9) below.

A central role in our construction is played by the supercovariant duality relation

$$
2 \mathcal{V}^{m}{ }_{A}\left(P^{\mu A}-\Gamma_{A \dot{A}}^{I} \bar{\psi}^{\mu I} \chi^{\dot{A}}\right)-\frac{i}{2} \mathcal{V}^{m}{ }_{I J} \Gamma_{\dot{A} \dot{B}}^{I J} \bar{\chi}^{\dot{A}} \gamma^{\mu} \chi^{\dot{B}},
$$

the gauged theory. The first step is the covariantization of derivatives in (3) according to

$$
\begin{aligned}
\mathcal{V}^{-1} \mathcal{D}_{\mu} \mathcal{V} & \equiv \mathcal{V}^{-1} \partial_{\mu} \mathcal{V}+g B_{\mu}{ }^{m} \mathcal{V}^{-1} t_{m} \mathcal{V} \\
& =\mathcal{P}_{\mu}^{A} Y^{A}+\frac{1}{2} \mathcal{Q}_{\mu}^{I J} X^{I J},
\end{aligned}
$$

with gauge coupling constant $g$, where we now use projected indices $m, n, \ldots$ for the gauge group. The nonAbelian field strength reads

$$
B_{\mu \nu}{ }^{m}=\partial_{\mu} B_{\nu}{ }^{m}-\partial_{\nu} B_{\mu}{ }^{m}+g f^{m}{ }_{n p} B_{\mu}{ }^{n} B_{\nu}{ }^{p} .
$$

The modified currents defined in (7) lead to a first $g$-dependent modification of the ungauged Lagrangian [5]

$$
\begin{aligned}
\mathcal{L}^{(0)}= & -\frac{1}{4} e R+\frac{1}{4} e \mathcal{P}^{\mu A} \mathcal{P}_{\mu}^{A}+\frac{1}{2} \epsilon^{\lambda \mu \nu} \bar{\psi}_{\lambda}^{I} D_{\mu} \psi_{\nu}^{I} \\
& -\frac{i}{2} e \bar{\chi}^{\dot{A}} \gamma^{\mu} D_{\mu} \chi^{\dot{A}}-\frac{1}{2} e \bar{\chi}^{\dot{A}} \gamma^{\mu} \gamma^{\nu} \psi_{\mu}^{I} \Gamma_{A \dot{A}}^{I} \dot{\mathcal{P}}_{\nu}^{A} .
\end{aligned}
$$

Next we observe that the non-Abelian extension of the duality relation (4) is obtained from this Lagrangian if we add the following CS term for the vector fields $B_{\mu}{ }^{m}$ :

$$
\mathcal{L}_{g}^{(1)}=-\frac{1}{4} g \epsilon^{\mu \nu \rho} B_{\mu}{ }^{m}\left(\partial_{\nu} B_{\rho m}+\frac{1}{3} g f_{m n p} B_{\nu}{ }^{n} B_{\rho}{ }^{p}\right) .
$$

This is very different from the situation in higher dimensions where the vector fields appear already in the 
ungauged theory via an Abelian kinetic term. By contrast, we find here that the CS term (10) induces a non-Abelian version of the duality relation (4) between scalar and vector fields; the latter is simply obtained by replacing the Abelian field strengths by (8), and the current $P_{\mu}^{A}$ by the one defined in (7), with no further modifications. This shows how the gauged theory brings in the dual vector fields, but without introducing new propagating degrees of freedom. However, because of the explicit appearance of the gauge fields on the right-hand side of the non-Abelian duality relation it is no longer possible to trade the vector fields for scalar fields and thereby eliminate them, unlike in [15].

The next steps in the construction are standard [2]: the $\mathcal{O}(g)$ terms in the currents necessitate extra bilinear fermionic terms in the Lagrangian

$$
\begin{aligned}
\mathcal{L}_{g}^{(2)}= & \frac{1}{2} g e A_{1}^{I J} \bar{\psi}_{\mu}^{I} \gamma^{\mu \nu} \psi_{\nu}^{J}+i g e A_{2}^{I \dot{A}} \bar{\chi}^{\dot{A}} \gamma^{\mu} \psi_{\mu}^{I} \\
& +\frac{1}{2} g e A_{3}^{\dot{A} \dot{B}} \bar{\chi}^{\dot{A}} \chi^{\dot{B}},
\end{aligned}
$$

as well as the following modifications of the fermionic variations:

$$
\delta_{g} \psi_{\mu}^{I}=i g A_{1}^{I J} \gamma_{\mu} \epsilon^{J}, \quad \delta_{g} \chi^{\dot{A}}=g A_{2}^{I \dot{A}} \epsilon^{I} .
$$

Here the $\mathrm{SO}(16)$ tensors $A_{1,2,3}$ depend on the scalar fields $\mathcal{V}$ in a way that remains to be specified and introduce Yukawa-type couplings between the scalars and the fermions beyond the derivative couplings generated by (3). At $\mathcal{O}\left(g^{2}\right)$, supersymmetry demands that we add the scalar potential

$$
\mathcal{L}_{g}^{(3)}=\frac{1}{8} g^{2} e\left(A_{1}^{I J} A_{1}^{I J}-\frac{1}{2} A_{2}^{I \dot{A}} A_{2}^{I \dot{A}}\right) .
$$

The check of local supersymmetry requires several identities that these tensors must obey. We list here only the most important ones. They are

$$
\begin{aligned}
\mathcal{V}^{m}{ }_{A} \Gamma_{A \dot{A}}^{(I} A_{2}^{J) \dot{A}} & =\mathcal{V}^{m}{ }_{I K} A_{1}^{J K}+\mathcal{V}^{m}{ }_{J K} A_{1}^{I K}, \\
\Gamma_{A \dot{A}}^{[I} A_{2}^{J]} & =\mathcal{V}^{C}{ }_{I J} \Theta_{C \mathcal{D}} \mathcal{V}^{\mathcal{D}}{ }_{A}, \\
\delta_{A B} A_{1}^{I I}+\Gamma_{A \dot{A}}^{I} A_{3}^{\dot{A} \dot{B}} \Gamma_{B \dot{B}}^{I} & =16 \mathcal{V}^{C}{ }_{A} \Theta_{C \mathcal{D}} \mathcal{V}^{\mathcal{D}}{ }_{B},
\end{aligned}
$$

with $\Gamma_{A \dot{A}}^{I} A_{2}^{I \dot{A}}=0$. In addition we have the differential relations

$$
\begin{aligned}
\mathcal{D}_{\mu} A_{1}^{I J}= & \mathcal{P}_{\mu}{ }^{A} \Gamma_{A \dot{A}}^{(I} A_{2}^{J) \dot{A}}, \\
\mathcal{D}_{\mu} A_{2}^{I \dot{A}}= & \frac{1}{2} \mathcal{P}_{\mu}{ }^{A}\left(\Gamma_{A \dot{B}}^{I} A_{3}^{\dot{A} \dot{B}}+\Gamma_{A \dot{A}}^{J} A_{1}^{I J}\right) \\
& -\frac{1}{2} \mathcal{P}_{\mu}{ }^{A} \Gamma_{B \dot{A}}^{I} \mathcal{V}^{\mathcal{A}}{ }_{A} \Theta_{\mathcal{A B}} \mathcal{V}^{\mathcal{B}}{ }_{B},
\end{aligned}
$$

and the quadratic identities

$$
\begin{gathered}
A_{1}^{I K} A_{1}^{K J}-\frac{1}{2} A_{2}^{I \dot{A}} A_{2}^{J \dot{A}}=\frac{1}{16} \delta^{I J}\left(A_{1}^{K L} A_{1}^{K L}-\frac{1}{2} A_{2}^{K \dot{A}} A_{2}^{K \dot{A}}\right) \\
3 A_{1}^{I J} A_{2}^{J \dot{A}}-A_{2}^{I \dot{B}} A_{3}^{\dot{A} \dot{B}}=\frac{1}{16} \Gamma_{A \dot{A}}^{I} \Gamma_{A \dot{B}}^{J}\left(3 A_{1}^{J K} A_{2}^{K \dot{B}}\right. \\
\left.-A_{2}^{J \dot{C}} A_{3}^{\dot{B}} \dot{C}\right) .
\end{gathered}
$$

Remarkably, the $\mathrm{SO}(16)$ representations of the tensors $A_{1,2,3}$ combine into a representation of $E_{8(8)}$ according to $135 \oplus \mathbf{1 8 2 0} \oplus \mathbf{1 9 2 0}=\mathbf{3 8 7 5}$, with the possibility of an extra singlet in the trace parts of $A_{1}^{I J}$ and $A_{3}^{A B}$. Apart from the extra singlet, this is analogous to the situation in $D=4$ and $D=5$, where the Yukawa couplings are given by tensors transforming in the 912 of $E_{7(7)}$ [16] and in the $\mathbf{3 5 1}$ of $E_{6(6)}$ [17], respectively.

Modulo higher order fermionic terms we can now establish that the full Lagrangian

$$
\mathcal{L}=\mathcal{L}^{(0)}+\mathcal{L}_{g}^{(1)}+\mathcal{L}_{g}^{(2)}+\mathcal{L}_{g}^{(3)}
$$

is indeed supersymmetric provided the tensors $A_{1,2,3}$ solve Eqs. (14)-(16). In the limit $g \rightarrow 0$ one reobtains the ungauged theory of Ref. [5]. Furthermore, the $N=16$ superalgebra closes in the usual way, except for an extra non-Abelian $G_{0}$-gauge transformation with parameter

$$
\Lambda^{m}=2 \mathcal{V}^{m}{ }_{I J} \bar{\epsilon}_{1}^{I} \epsilon_{2}^{J}+i B_{\mu}{ }^{m} \bar{\epsilon}_{1}^{I} \gamma^{\mu} \epsilon_{2}^{I} .
$$

The main task is now to ascertain that the relations (14)-(16) do admit nontrivial solutions and to determine the possible gauge groups $G_{0}$. Any subgroup $G_{0} \subset E_{8(8)}$ is characterized by its embedding tensor $\Theta_{\mathcal{A B}}$ [cf. (5)] which decomposes as

$$
\Theta_{\mathcal{A B}}=\theta \eta_{\mathcal{A B}}+\Theta_{\mathcal{A B}}^{3875}+\Theta_{\mathcal{A B}}^{27000},
$$

with the (indefinite) $E_{8(8)}$ Cartan-Killing metric $\eta_{\mathcal{A B}}$, in accordance with the symmetric $E_{8(8)}$ tensor product

$$
(248 \times 248)_{\mathrm{sym}}=1+3875+27000 .
$$

Guided by [2] we define the $T$ tensor by

$$
T_{\mathcal{A} \mid \mathcal{B}} \equiv \mathcal{V}^{C}{ }_{\mathcal{A}} \mathcal{V}^{\mathcal{D}}{ }_{\mathcal{B}} \Theta_{C \mathcal{D}}
$$

and note that Eqs. (14) define the $\mathrm{SO}(16)$ tensors $A_{1,2,3}$ as functions of $T_{\mathcal{A} \mid \mathcal{B}}$ [unlike the cubic expressions in [2] and [17], the $T$ tensor (21) is quadratic in $\mathcal{V}$ due to (1)]. As the tensors $A_{1,2,3}$ combine into the $\mathbf{3 8 7 5}(+\mathbf{1})$ representation(s) of $E_{8(8)}$, Eqs. (14)-(16) can only be consistent if the 27000 is absent in (21), which in turn requires $\Theta_{\mathcal{A B}}$ to satisfy the condition

$$
\Theta_{\mathcal{A B}}^{27000}=0 \Longleftrightarrow \Theta_{\mathcal{A B}}=\theta \eta_{\mathcal{A B}}+\Theta_{\mathcal{A B}}^{3875} .
$$

If (22) is satisfied, the tensors $A_{1,2,3}$ which solve Eqs. (14)-(16) are given by

$$
\begin{aligned}
A_{1}^{I J} & =-\theta \delta_{I J}-\frac{1}{7} \mathcal{V}^{C}{ }_{I K} \mathcal{V}^{\mathcal{D}}{ }_{K J} \Theta_{C \mathcal{D}}^{3875}, \\
A_{2}^{I \dot{A}} & =-\frac{1}{7} \Gamma_{A \dot{A}}^{J} \mathcal{V}^{C}{ }_{I J} \mathcal{V}^{\mathcal{D}}{ }_{A} \Theta_{C \mathcal{D}}^{\mathbf{3 8 7 5}}, \\
A_{3}^{\dot{A} \dot{B}} & =2 \theta \delta_{\dot{A} \dot{B}}+\frac{1}{48} \Gamma_{\dot{A} \dot{B}}^{I J K L} \mathcal{V}^{C}{ }_{I J} \mathcal{V}^{\mathcal{D}}{ }_{K L} \Theta_{C \mathcal{D}}^{\mathbf{3 8 7 5},}
\end{aligned}
$$

as can be shown by a rather lengthy computation which relies in particular on the form of the projectors onto the irreducible parts of its tensor products [13]. Thus, (22) encodes the complete set of consistency equations for the gauged theory, thereby reducing the differential and bilinear identities for the a priori unknown tensors $A_{1,2,3}$ to a single linear algebraic equation for the embedding tensor $\Theta_{\mathcal{A B}}$. The formulation of this equation as a projection condition allows one to select the admissible gauge subgroups 
of $E_{8(8)}$ by purely group theoretical arguments. We do not aim for a complete classification of the solutions of (22) here, but rather discuss the most important examples.

The maximal compact subgroup of $E_{8(8)}$ satisfying the condition (22) is

$$
\mathrm{SO}(16) \supset G_{0}=\mathrm{SO}(8) \times \mathrm{SO}(8),
$$

where the ratio of coupling constants of the two factors is $g_{1} / g_{2}=-1$ such that the trace part $\theta$ of $\Theta_{\mathcal{A B}}$ vanishes. The embedding tensor for $\mathrm{SO}(8) \times \mathrm{SO}(8)$ is explicitly given by

$$
\begin{aligned}
& \Theta_{I J, K L}=\delta_{I[K} \varepsilon_{L] J}, \quad \Theta_{I J, A}=0=\Theta_{A, B},
\end{aligned}
$$

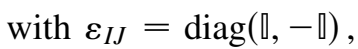

with a $16 \rightarrow 8+8$ split of the indices $I, J$. This tensor transforms in the $\mathbf{1 3 5}$ under $\mathrm{SO}(16)$ and automatically satisfies (22), because there is no $\mathbf{1 3 5}$ in the $\mathbf{2 7 0 0 0}$ of $E_{8(8)}$. By contrast, no $\mathrm{SO}(9)$ subgroup of $\mathrm{SO}(16)$ satisfies (22), in agreement with the fact that there is no $S^{8}$ compactification of $D=11$ supergravity to three dimensions. Likewise, it may be shown that apart from (24) none of its maximal compact subgroups of type $\mathrm{SO}(p) \times \mathrm{SO}(16-p)$ satisfies (22).

Among the maximal subgroups of $E_{8(8)}$, we have found three possible noncompact gauge groups

$$
\begin{aligned}
& G_{0}=E_{7(-5)} \times \mathrm{SU}(2) \quad \text { with }\left(g_{\mathrm{SU}(2)} / g_{E_{7}}\right)=-3, \\
& G_{0}=E_{6(-14)} \times \mathrm{SU}(3) \quad \text { with }\left(g_{\mathrm{SU}(3)} / g_{E_{6}}\right)=-2, \\
& G_{0}=F_{4(-20)} \times G_{2} \quad \text { with }\left(g_{G_{2}} / g_{F_{4}}\right)=-3 / 2,
\end{aligned}
$$

all of which have nonvanishing trace $\theta$. A further and "extremal" solution to (22) is $G_{0}=E_{8(8)}$ with $\Theta_{\mathcal{A B}}=$ $\theta \eta_{\mathcal{A B}}$.

An immediate question concerns the possible existence of $\mathrm{AdS}_{3}$ ground states with additional symmetries. The fact that $d=11$ supergravity does not appear to admit compactifications of this type would seem to indicate that the potential (13) does not possess fully supersymmetric stationary points. Inspection of the supersymmetry variations (2) and (12) reveals that a $(16,0)$ invariant $\mathrm{AdS}_{3}$ ground state can only exist if $A_{1}^{I J} \propto \delta_{I J}$, i.e., if the trace of $\Theta_{\mathcal{A B}}$ does not vanish. The $\mathrm{SO}(8) \times \mathrm{SO}(8)$ gauged theory (24) has a maximally symmetric ground state characterized by $\mathcal{V}=\rrbracket_{248}$, which by (23) implies

$$
A_{1}^{I J}=-\frac{1}{7} \varepsilon_{I J}, \quad A_{2}^{I \dot{A}}=0, \quad A_{3}^{\dot{A} \dot{B}}=0,
$$

with $\varepsilon_{I J}$ from (25). The Lagrangian (17) then reduces to the $(8,8)$ CS theory of [8] and reproduces the vacuum found there. On the other hand, the truncation of the gauge group $\mathrm{SO}(8) \times \mathrm{SO}(8)$ to its diagonal subgroup is presumably related to the theory obtained by direct reduc- tion of the $D=4$ gauged theory, which no longer exhibits an $\mathrm{SO}(8)$ invariant AdS but rather a domain wall ground state [9]. Pursuing an analysis of the full theory along the lines of [18] should allow one to understand in detail the presumed relation to the matrix string theory [7] on the boundary.

Finally, dimensional reduction of the gauged theories (17) yields maximal gauged supergravities in two dimensions as well. In this reduction, the vector fields $B_{\mu}{ }^{m}$ give rise not only to two-dimensional vectors, but also to the dual scalar fields at the bottom of the infinite tower of dual potentials generated by the $E_{9(9)}$ symmetry of $N=16, D=2$ supergravity. It would be most interesting to see whether our construction can be extended to yield gauged supergravities in two dimensions with infinitedimensional gauge groups.

A detailed account of our results will be presented elsewhere. This work was supported in part by the European Union under Contract No. HPRN-CT-2000-00122.

*UMR 8549: Unité Mixte du Centre National de la Recherche Scientifique, et de l'Ecole Normale Supérieure.

[1] O. Aharony et al., Phys. Rep. 323, 183 (2000).

[2] B. de Wit and H. Nicolai, Nucl. Phys. B208, 323 (1982).

[3] Supergravities in Diverse Dimensions, edited by A. Salam and E. Sezgin (World Scientific, Singapore, 1989).

[4] B. Julia, in Unified Field Theories in More Than 4 Dimensions Including Exact Solutions, edited by V.D. Sabbata and E. Schmutzer (World Scientific, Singapore, 1983), pp. 215-236.

[5] N. Marcus and J. Schwarz, Nucl. Phys. B228, 145 (1983).

[6] E. Cremmer, B. Julia, and J. Scherk, Phys. Lett. 76B, 409 (1978).

[7] R. Dijkgraaf, E. Verlinde, and H. Verlinde, Nucl. Phys. B500, 43 (1997).

[8] A. Achúcarro and P. K. Townsend, Phys. Lett. B 180, 89 (1986).

[9] H. Lu, C. N. Pope, and P. K. Townsend, Phys. Lett. B 391, 39 (1997).

[10] M. Cvetič, H. Lu, and C. N. Pope, Phys. Rev. D 62, 064028 (2000).

[11] N.S. Deger, A. Kaya, E. Sezgin, and P. Sundell, Nucl. Phys. B573, 275 (2000).

[12] C. M. Hull, Phys. Lett. 142B, 39 (1984).

[13] K. Koepsell, H. Nicolai, and H. Samtleben, J. High Energy Phys. 4, 023 (1999).

[14] K. Koepsell, H. Nicolai, and H. Samtleben, Classical Quantum Gravity 17, 3689 (2000).

[15] E. Cremmer, B. Julia, H. Lu, and C. N. Pope, Nucl. Phys. B523, 73 (1998).

[16] B. de Wit and H. Nicolai, Nucl. Phys. B231, 506 (1984).

[17] M. Günaydin, L. J. Romans, and N. P. Warner, Nucl. Phys. B272, 598 (1986).

[18] D. Z. Freedman, S. S. Gubser, K. Pilch, and N. P. Warner, Adv. Theor. Math. Phys. 3, No. 2 (1999). 